\title{
NÍVEL DE DANO, PLANTAS INVASORAS HOSPEDEIRAS, INIMIGOS NATURAIS E CONTROLE DO PSILÍDEO DA GOIABEIRA (Triozoida sp.) NO SUBMÉDIO SÃO FRANCISCO ${ }^{1}$
}

\author{
FLÁVIA RABELO BARBOSA², RACHEL GONÇALVES FERREIRA³, LÚCIA HELENA PIEDADE KIILL², EDUARDO \\ ALVES DE SOUZA4 ${ }^{4}$, WELLINGTON ANTONIO MOREIRA², JOSÉ ADALBERTO DE ALENCAR ${ }^{5}$, FRANCISCA \\ NEMAURA PEDROSA HAJI ${ }^{2}$
}

\begin{abstract}
RESUMO - Com o objetivo de obter informações para implementação do Manejo Integrado do psilídeo da goiabeira, no Vale do São Francisco, realizaram-se estudos sobre nível de dano, plantas invasoras hospedeiras, seletividade e efeito do thiamethoxam 10GR e $250 \mathrm{WG}$ no controle de Triozoida sp. O experimento foi conduzido em Petrolina-PE, em blocos ao acaso, com quatro repetições. Os tratamentos foram: 1) thiamethoxam 10GR aplicado no solo; 2) thiamethoxam 250WG pulverizado semanalmente; 3) thiamethoxam 10GR aplicado no solo + thiamethoxam $250 \mathrm{WG}$ pulverizado semanalmente; 4, 5 e 6) pulverizações com thiamethoxam $250 \mathrm{WG}$ quando se constatou 10, 20 e 30\% de ramos infestados, respectivamente; 7, 8 e 9) thiamethoxam 10GR aplicado no solo + thiamethoxam 250WG pulverizado semanalmente quando se constatou 10,20 e $30 \%$ de ramos danificados, respectivamente e 10) testemunha (sem inseticida). A percentagem de galhos infestados na testemunha (33,6\%) diferiu significativamente dos tratamentos $2(2,8 \%), 3(4,33 \%), 4(19,7 \%), 7(13,45 \%), 8(14,50 \%)$ e $9(15,00 \%)$. Nas parcelas tratadas com thiamethoxam, a redução populacional de inimigos naturais variou de 12,5 a 39,6\%, correspondendo às notas 1 e 2 na escala de seletividade $(1=$ atóxico $(<25 \%), 2=$ pouco tóxico $(25-50 \%)$. $\mathrm{O}$ número e o peso de frutos foram semelhantes em todos os tratamentos. Foram encontradas 51 espécies de invasoras, nenhuma hospedeira do psilídeo.
\end{abstract}

Termos para indexação: Psidium guajava, manejo integrado de pragas (MIP), seletividade.

\section{STUDIES OF DAMAGE LEVEL, WEED HOST PLANTS, NATURAL ENEMIES AND CONTROL OF Triozoida sp. IN GUAVA PLANTS AT THE SÃO FRANCISCO RIVER VALLEY}

\begin{abstract}
This study was conducted to increment the integrated management of Triozoida sp. (Hemiptera, Psylliidae) in guava plants at the São Francisco River Valley. The damage level, weed hosts, selectivity and effect of the thiamethoxam 10GR and 250WG in the control of psylliidae were evaluated. The experiment was carried out in an irrigated area, at Petrolina, Pernambuco, in a randomized block design with four replications. Treatments consisted of: 1) thiamethoxam 10GR applied on soil; 2) thiamethoxam 250WG sprayed weekly; 3) thiamethoxam 10GR applied on soil + thiamethoxam 250WG sprayed weekly; 4; 5; 6) thiamethoxam $250 \mathrm{WG}$ sprayed when 10, 20 and 30\% of infested branches were reached, respectively; $7 ; 8 ; 9)$ thiamethoxam 10GR applied on soil + thiamethoxam 250WG sprayed when 10, 20 and 30\% of infested branches were reached, respectively; and 10) control (no insecticide). The percentage of infested branches in the control treatment differed significantly from the treatments $2(2.8 \%), 3$ (4.3\%), $4(19.7 \%), 7(13.4 \%), 8(14.5 \%)$ and $9(15.0 \%)$. When thiamethoxam was used, the population reduction of natural enemies ranged from 12.5 to $39.6 \%$, corresponding, in the selective scale, to grades $(1=$ non offensive $(<25 \%), 2=$ not very toxic $(25-50 \%)$. Number and weight of fruits were similar in all the treatments. Fifty one weed species and no host of Triozoida sp. were found.
\end{abstract}

Index terms: Psidium guajava, integrated pest management (IPM), selectivity.

\section{INTRODUÇÃO}

A área explorada com a cultura da goiabeira no Brasil cresce intensivamente, sendo o Estado de Pernambuco o segundo maior produtor nacional (Agrianual, 2000). A alteração do agroecossistema, provocada pela expansão desta cultura, tem propiciado condições favoráveis ao surgimento de problemas fitossanitários, destacando-se, dentre estes, os relacionados às pragas.

No Brasil, Maricone \& Soubihe Sobrinho (1961) registraram mais de cem espécies de insetos em goiabeira. No Submédio São Francisco, o psilídeo Triozoida sp. (Hemiptera: Psyllidae) é a principal praga da cultura (Barbosa, 2001a). A partir de 1995, a praga, antes desconhecida na região, vem ocasionando severos danos, em decorrência da redução da área foliar, impedindo o desenvolvimento das brotações e, conseqüentemente, comprometendo a produção. A presença de Triozoida $\mathrm{sp}$. em goiabeira foi também relatada nos estados de São Paulo, Maranhão, Rio de Janeiro e Paraná (Nakano \& Silveira Neto, 1968; Lemos, 2000; Souza et al., 2000; Menezes Júnior \& Pasini, 2001).
No contexto da filosofia do Programa de Manejo Integrado de Pragas, o uso de inseticidas constitui-se em uma das táticas disponíveis para o agricultor regular as populações de insetos potencialmente danosos à cultura. Contudo, torna-se importante determinar o nível de dano, o impacto dos inseticidas sobre os inimigos naturais e o papel de plantas infestantes como hospedeiras alternativas da praga ou como abrigo para os inimigos naturais. O objetivo deste trabalho foi estudar o nível de dano, plantas invasoras hospedeiras e verificar a seletividade e o efeito do thiamethoxam, em duas formulações, no controle do psilídeo Triozoida sp., em goiabeira.

\section{MATERIALEMÉTODOS}

O experimento foi conduzido no período de março a novembro de 2001, em pomar comercial, no município de Petrolina-PE. Utilizaramse plantas da cultivar Paluma com 24 meses de idade, no espaçamento $5 \times 4 \mathrm{~m}$. Os tratamentos foram iniciados após a poda das plantas, no início da brotação. Os tratamentos foram: 1) thiamethoxam 10GR aplicado no solo; 2) thiamethoxam $250 \mathrm{WG}$ pulverizado semanalmente; 3 )

\footnotetext{
${ }^{1}$ (Trabalho 022/2003). Recebido: 06/02/2003. Aceito para publicação: 24/10/2003.

${ }^{2}$ Eng. Agr., D.Sc., Pesquisador da Embrapa Semi-Árido, BR 428, km 156, C.P. 23, CEP 56302-970, Petrolina-PE. Fone: (087) 3862-1711. E-mail: flavia@cpatsa.embrapa.br.

${ }^{3}$ Eng. Agr., D.Sc., Pesquisador da Empresa Pernambucana de Pesquisa Agropecuária-IPA, C.P. 1022, CEP 50761-000, Recife-PE. Fone (081) 3325-5988. E-mail: rachelgferreira@ig.com.br.

${ }^{4}$ Estagiário Embrapa Semi-Árido.

${ }^{5}$ Eng. Agr., M.Sc., Pesquisador da Embrapa Semi-Árido, BR 428, km 156, C.P. 23, CEP 56302-970, Petrolina-PE. Fone (087) 3862-1711. E-mail: alencar@cpatsa.embrapa.br.
} 
thiamethoxam 10GR aplicado no solo + thiamethoxam 250WG pulverizado semanalmente; 4, 5 e 6) pulverizações com thiamethoxam $250 \mathrm{WG}$ quando se constatou 10,20 e $30 \%$ de ramos infestados, respectivamente; 7,8 e 9) thiamethoxam $10 \mathrm{GR}$ aplicado no solo + thiamethoxam $250 \mathrm{WG}$ pulverizado semanalmente quando se constatou 10,20 e $30 \%$ de ramos infestados, respectivamente e 10) testemunha (sem inseticida). Foi utilizado $75 \mathrm{~g}$ p.c./planta de thiamethoxam $10 \mathrm{GR}$ e 20 g p.c./100 litros d'água de thiamethoxam 250WG. A aplicação de thiamethoxam 10GR, independente do tratamento, foi realizada apenas uma vez, manualmente, em sulcos, em ambos os lados da fileira das plantas, na projeção da copa. As pulverizações foram realizadas com pulverizador costal manual, gastando-se em média $500 \mathrm{~mL}$ de calda por planta.

O delineamento experimental foi o de blocos ao acaso com quatro repetições, sendo cada tratamento constituído por doze plantas. Após a primeira aplicação dos inseticidas, as amostragens do número de ramos danificados por psilídeos e de inimigos naturais foram realizadas em intervalo semanal, caminhando-se ao redor da planta e anotando-se o número de ramos infestados, bem como, o número e espécies de inimigos naturais presentes. Foram também registrados o número e peso médio de frutos produzidos por planta/tratamento. As porcentagens de redução populacional dos inimigos naturais foram mensuradas comparando-se a média do número de inimigos naturais nas plantas testemunhas com a média do número de inimigos naturais nas plantas nos tratamentos, as quais foram enquadradas na seguinte escala de seletividade: $1=$ atóxico $(<25 \%) ; 2=$ pouco tóxico $(25-50 \%)$; 3 = moderadamente tóxico $(51-75 \%)$ e $4=$ tóxico $(>75 \%)$ de redução populacional de inimigos naturais (Hassan, 1994).

Para se constatar a presença-ausência de psilídeos e inimigos naturais nas plantas invasoras presentes no interior e nas bordaduras do pomar, foram realizadas amostragens com rede entomológica ou coletadas partes infestadas da planta hospedeira.

O levantamento das plantas invasoras foi realizado por meio de amostragens aleatórias em cinco parcelas de $30 \mathrm{~m}^{2}$, de acordo com a determinação da área mínima (Kiill \& Lima, 2001).

Para a análise de variância, os dados referentes à percentagem de galhos infestados foram transformados em arco seno $\sqrt{x}$. As médias foram comparadas pelo teste de Tukey, ao nível de $5 \%$ de probabilidade.

\section{RESULTADOS E DISCUSSÃO}

A percentagem de galhos com brotações infestadas na testemunha foi de $33,62 \%$, diferindo significativamente dos resultados dos tratamentos 2, 3, 4, 7, 8 e 9, com, respectivamente, 2,83\%; 4,33\%; $19,71 \% ; 13,45 \% ; 14,50 \%$ e $15,00 \%$. Não foi constatada redução significativa na porcentagem de galhos infestados quando se utilizou somente thiamethoxam 10GR, isto é, aplicação do produto no solo; bem como quando foram realizadas pulverizações com thiamethoxam $250 \mathrm{WG}$, quando foram observados $20 \%$ e $30 \%$ de galhos infestados pelo psilídeo. Também não foram constatadas diferenças significativas entre os tratamentos 2 e 3 , assim como entre 3 e 7, entre 4, 5, 6 e, ainda, entre 6, 7, 8, 9 e 3, 7, 8 e 9 (Tabela 1 ).

A eficiência de inseticidas da classe dos neonicotinóides, no controle do psilídeo da goiabeira, foi também observada por Barbosa et al. (1999, 2001b), que observaram redução significativa dos danos desta praga, quando utilizaram imidacloprid e thiacloprid, em pulverização.

Observou-se que o número e o peso de frutos foram semelhantes em todos os tratamentos. A testemunha (sem inseticida), que apresentou $33,62 \%$ de galhos infestados, produziu $27,42 \mathrm{~kg}$, não diferindo, respectivamente, dos tratamentos 2 e 3 , que produziram 33,42 e 36,59 kg, com menos de 5\% de galhos infestados (Tabela 1). Tais resultados indicam que o nível de controle da praga para a região é de $30 \%$ de galhos infestados, desde que, a goiabeira pode suportar até $30 \%$ de galhos danificados pelo psilídeo, sem que haja dano econômico, não havendo, portanto, necessidade de pulverizações semanais, como normalmente o produtor realiza.

Em relação aos inimigos naturais, constatou-se a presença dos coccinelídeos Cycloneda sanguinea, Eriopis conexa e Scymnus sp. e de espécimes de crisopídeos (Crysoperla externa e Ceraechrysa cubana), aracnídeos, sirfídeos, nabídeos e tacnídeos. O número médio de inimigos naturais na testemunha foi 10,30 por planta, enquanto nas plantas tratadas, houve variação de 6,06 a 8,78 , correspondendo às notas 2 e 1 , na escala de seletividade (Tabela 1 ), ou seja, 39,6\% a $12,5 \%$ de redução populacional de inimigos naturais, em relação às parcelas não tratadas.

$\mathrm{Na}$ área estudada foram encontradas 51 espécies de plantas invasoras pertencentes a 38 gêneros e 15 famílias botânicas (Tabela 2), contudo, nenhuma espécie foi hospedeira do psilídeo. Na Tabela 2 estão relacionadas as espécies invasoras inventariadas, com seus respectivos nomes científicos e vulgar, família botânica e freqüência. A família Poaceae foi a mais representativa, abrangendo $17,50 \%$ do total de espécies levantadas.

TABELA 1 - Efeito dos tratamentos na percentagem de ramos infestados por psilídeos, na população de inimigos naturais e no número e peso de frutos de goiaba. Petrolina-PE, 2001.

\begin{tabular}{|c|c|c|c|c|c|}
\hline \multirow[b]{2}{*}{ Tratamento } & \multirow{2}{*}{$\begin{array}{c}\text { Galhos } \\
\text { infestados } 1(\%)\end{array}$} & \multirow{2}{*}{$\begin{array}{l}\text { Inimigos naturais } \\
\left(\mathrm{n}^{\mathrm{o}} / \text { planta }\right)\end{array}$} & \multirow{2}{*}{$\begin{array}{l}\text { Seletividade } \\
\text { (Nota) }^{2}\end{array}$} & \multicolumn{2}{|c|}{ Frutos/planta } \\
\hline & & & & $\left(n^{\underline{o}}\right)$ & Peso $(\mathrm{kg})$ \\
\hline 1. Thiamethoxam 10GR & $29,67 \mathrm{ab}$ & 7,89 & 1 & $200,25 \mathrm{a}$ & $33,30 \mathrm{a}$ \\
\hline 2. Thiamethoxam 250WG, pulverizações semanais & $2,83 \mathrm{e}$ & 8,53 & 1 & $201,00 \mathrm{a}$ & $33,42 \mathrm{a}$ \\
\hline $\begin{array}{l}\text { 3. Thiamethoxam } 10 \mathrm{GR}+\text { thiamethoxam } 250 \mathrm{WG} \text {, pulverizações } \\
\text { semanais }\end{array}$ & $4,33 \mathrm{de}$ & 6,06 & 2 & $206,25 \mathrm{a}$ & $36,59 \mathrm{a}$ \\
\hline 4. Thiamethoxam $250 \mathrm{WG}, 10 \%$ ramos infestados & $19,71 \mathrm{bc}$ & 8,69 & 1 & $218,75 \mathrm{a}$ & $34,30 \mathrm{a}$ \\
\hline 5. Thiamethoxam $250 \mathrm{WG} / 20 \%$ ramos infestados & $23,75 \mathrm{ab}$ & 7,97 & 1 & $176,75 \mathrm{a}$ & $29,40 \mathrm{a}$ \\
\hline 6. Thiamethoxam $250 \mathrm{WG} / 30 \%$ de ramos infestados & $28,46 a b c$ & 8,78 & 1 & $186,25 \mathrm{a}$ & $30,97 \mathrm{a}$ \\
\hline $\begin{array}{l}\text { 7. Thiamethoxam } 10 \mathrm{GR}+\text { thiamethoxam } 250 \mathrm{WG} / 10 \% \text { ramos } \\
\text { infestados }\end{array}$ & $13,45 \mathrm{~cd}$ & 8,31 & 1 & $219,75 \mathrm{a}$ & $36,37 \mathrm{a}$ \\
\hline $\begin{array}{l}\text { 8. Thiamethoxam } 10 \mathrm{GR}+\text { thi amethoxam } 250 \mathrm{WG} / 20 \% \text { ramos } \\
\text { infestados }\end{array}$ & $14,50 \mathrm{~cd}$ & 7,83 & 1 & $201,25 \mathrm{a}$ & $33,45 \mathrm{a}$ \\
\hline $\begin{array}{l}\text { 9. Thiamethoxam } 10 \mathrm{GR}+\text { thiamethoxam } 250 \mathrm{WG} / 30 \% \text { ramos } \\
\text { infestados }\end{array}$ & $15,00 \mathrm{~cd}$ & 7,61 & 1 & $194,50 \mathrm{a}$ & $32,35 \mathrm{a}$ \\
\hline 10. Testemunha & $33,62 \mathrm{a}$ & 10,03 & - & $165,00 \mathrm{a}$ & $27,42 \mathrm{a}$ \\
\hline
\end{tabular}

${ }^{1}$ Médias seguidas da mesma letra, na mesma coluna, não diferem entre si, pelo teste de Tukey a $5 \%$ de probabilidade;

${ }^{2} 1=$ atóxico $(<25 \%)$; $2=$ pouco tóxico (25-50\%); 3=moderadamente tóxico (51-75\%); 4=tóxico ( $\left.>75 \%\right)$ de redução populacional dos inimigos naturais (Hassan, 1994): coccinelídeos (Cycloneda sanguinea, Eriopis conexa e Scymnus sp.), crisopídeos (Crysoperla externa e Ceraechrysa cubana), aracnídeos, sirfídeos, tacnídeos e nabídeos. 
TABELA 2 - Espécies invasoras inventariadas em pomar de goiabeira, no Submédio São Francisco, com seus respectivos nomes científicos e vulgares, família botânica e freqüência. Petrolina-PE, 2001.

\begin{tabular}{|c|c|c|}
\hline Família/Espécie & Nome Vulgar & Frequência (\%) \\
\hline \multicolumn{3}{|l|}{ Amaranthaceae } \\
\hline Althernanthera pungens H.B.K. & Falso pega-pinto & 20 \\
\hline \multicolumn{3}{|l|}{ Asteraceae } \\
\hline Acanthospermum hispidum DC. & Juiz de paz & 40 \\
\hline Bidens pilosa $L$. & Agulha & 20 \\
\hline Centratherum punctatum Cass. & Pincel roxo & 20 \\
\hline Eclipta alba Hassk. & Mato d'agua & 60 \\
\hline Emilia sonchifolia (L.) DC. & Serralha vermelha & 40 \\
\hline $\begin{array}{l}\text { Galinsoga parviflora Cav. } \\
\text { Convolvulaceae }\end{array}$ & Convolvulaceae & 20 \\
\hline Jacquemontia agrestis (Choisy) Meisn. & Cipó da flor azul & 20 \\
\hline \multicolumn{3}{|l|}{ Curcubitaceae } \\
\hline \multicolumn{3}{|l|}{ Euphorbiaceae } \\
\hline Chamaesyce hyssopifolia (L.) Small. & Falso quebra-pedra & 80 \\
\hline Chamaesyce hirta (L.) Millsp. & Orelha de mexirra & 60 \\
\hline \multicolumn{3}{|l|}{ Lamiaceae } \\
\hline $\begin{array}{c}\text { Marsypianthes chamaedrys (Vahl) Kuntz } \\
\text { Leguminosae }\end{array}$ & Leguminosae & 60 \\
\hline Caesalpinoidae & & \\
\hline Senna occidentalis (L.) Link & Fedegoso & 20 \\
\hline Senna tora (L.) Roxb. & Mata pasto liso & 60 \\
\hline \multicolumn{3}{|l|}{ Papilionoidae } \\
\hline Indigofora hirsuta $L$ & Bananinha 5 folhas & 60 \\
\hline Centrosema brasilianum (L.) Benth & Feijão flor roxa & 20 \\
\hline Crotalaria incana $L$. & Guiso de cascavel & 20 \\
\hline Crotalaria retusa $L$. & Crotalaria & 20 \\
\hline Phaseolus nathyroides $L$ & Feijão de rolinha & 100 \\
\hline $\begin{array}{l}\text { Desmodium tortuosum (Sw.) DC. } \\
\text { Mimosoidae }\end{array}$ & Rapadura de cavalo & 60 \\
\hline Mimosa tenuiflora (Willd.) Poir. & Jurema preta & 20 \\
\hline Mimosa arenosa (Willd.) Poir & Jurema vermelha & 20 \\
\hline \multicolumn{3}{|l|}{ Malvaceae } \\
\hline Herissanthia crispa (L.) rizicky & Malva rasteira & 100 \\
\hline Pavonia cancellata Cav. & Corda de viola 1 & 60 \\
\hline Sida cordifolia $L$ & Malva grossa & 100 \\
\hline Wissadula peritlocifolia & Quatro sementes & 40 \\
\hline Sida santhamaranthensis $H$. Monteiro & Reloginho 2 & 40 \\
\hline \multicolumn{3}{|l|}{ Nyctaginaceae } \\
\hline \multicolumn{3}{|l|}{ Onagraceae } \\
\hline \multicolumn{3}{|l|}{ Poaceae } \\
\hline Cenchrus ciliaris L. & Capim buffel & 60 \\
\hline Cenchrus echinatus $L$. & Capim carrapicho & 100 \\
\hline Chloris polydactyla (L.) Sw. & Capim marrom & 100 \\
\hline Dactyloctenium aegyptium (L.) Beauv. & Capim pé papagaio & 80 \\
\hline Digitaria horizontalis Willd. & Capim fino & 100 \\
\hline Eleusine indica (L.) Gaertn. & Capim pé-de-galinha & 20 \\
\hline Digitaria insularis (L.) Mez ex. Ekman & Capim amargoso & 60 \\
\hline Rhynchelytrum repens (Willd.)C.E. Hubb. & Capim favorito & 80 \\
\hline $\begin{array}{c}\text { Digitaria bicornis (Lam.) Roem. \& Schult. } \\
\text { Portulacaceae }\end{array}$ & Capim sempre verde & 40 \\
\hline $\begin{array}{l}\text { Portulaca oleracea } L . \\
\text { Rubiaceae }\end{array}$ & Beldroega de porco & 60 \\
\hline Richardia grandiflora (Cham. \& Shlecht.) Steudel & Ervanço branco & 80 \\
\hline $\begin{array}{l}\text { Diodia teres Walt. } \\
\text { Solanaceae }\end{array}$ & Ervanço preto & 40 \\
\hline $\begin{array}{l}\text { Solanum viarum Dun. } \\
\text { Sterculiaceae }\end{array}$ & Melancia da praia & 60 \\
\hline Waltheria $s p$ & Malva flor amarela 2 & 100 \\
\hline $\begin{array}{l}\text { Waltheria rotundifolia Schrank } \\
\text { Sem identificacão }\end{array}$ & Malva prateada & 100 \\
\hline Não identificada 1 & Desconhecida & 20 \\
\hline
\end{tabular}


Nas plantas invasoras, observou-se crisopídeos (C. externa e C. cubana), principalmente, sobre espécies de poáceas. Ovos e larvas destes insetos foram observados também na goiabeira e em outras invasoras. Outros insetos predadores como sirfídeos, nabídeos e coccinelídeos foram encontrados com freqüência sobre plantas invasoras. Adultos de sirfídeos foram encontrados, principalmente, sobre Herissanthia crispa (L.) Brizicky e Bidens pilosa L. Coccinelídeos e sirfídeos foram encontrados predando pulgões em diversas invasoras. A presença de invasoras na área em estudo possibilitou a multiplicação destes insetos, que auxiliam na manutenção do equilíbrio populacional do psilídeo da goiabeira. Contudo, estudos adicionais sobre o manejo de invasoras necessitam ser realizados, para que não se tornem competidoras da cultura. De acordo com Altieri (1991) o aumento da diversidade de espécies vegetais pode resultar na diminuição de algumas pragas, devido ao aumento da diversidade e abundância de inimigos naturais.

A demanda por uma solução a curto prazo, para atender as necessidades dos produtores de compatibilização do cultivo da goiabeira com requisitos de ordem econômica, ecológica e social, expressa a necessidade urgente de tecnologias sustentáveis, próprias para as condições do Semi-Árido. Os conhecimentos gerados neste trabalho fornecerão subsídios para o desenvolvimento do Manejo Integrado de Pragas da goiabeira no Vale do Rio São Francisco.

\section{CONCLUSÕES}

1) As menores percentagens de galhos infestados pelo psilídeo da goiabeira foram observadas nos tratamentos: thiamethoxam $250 \mathrm{WG}$ em pulverizações semanais, thiamethoxam 10 GR no sulco + pulverizações semanais;

2) Thiamethoxam $10 \mathrm{GR}$ e thiamethoxam $250 \mathrm{WG}$ apresentam boa seletividade para coccinelídeos, crisopídeos, aracnídeos, sirfídeos e tacnídeos;

3) A goiabeira pode suportar até $30 \%$ de galhos infestados pelo psilídeo, sem que haja redução significativa no número e peso de frutos;

4) As 51 espécies de plantas invasoras pertencentes ao agroecossistema estudado não são hospedeiras do psilídeo da goiabeira;

5) As plantas invasoras encontradas no agroecossistema estudado abrigam inimigos naturais do psilídeo.

\section{REFERÊNCIAS BIBLIOGRÁFICAS}

AGRIANUAL 2001: Anuário de agricultura brasileira. São Paulo: FNP Consultoria e Comércio, 2002. p.364-369.

ALTIERI, M.A. How best can we use biodiversity in agroecosystem. Outlook on agriculture. Wallingsord, v.20, p.15-23, 1991.

BARBOSA, F.R. Direto na seiva. Cultivar-Hortaliças e Frutas, n. 8, p.27-28, 2001.

BARBOSA, F.R.; SOUZA E.A. de; SIQUEIRA, K.M.M. de; MOREIRA, W.A.; ALENCAR, J.A. de; HAJI, F.N.P. Eficiência e seletividade de inseticidas no controle do psilídeo (Triozoida sp.), em goiabeira. Pesticidas - Revista de Ecotoxicologia e Meio Ambiente, Curitiba, v. 11, p.45-52, 2001.

BARBOSA, F.R.; SANTOS, A.P. dos; HAJI, A.T.; MOREIRA, W.A.; HAJI, F.N.P.; ALENCAR, J.A. de. Eficiência e seletividade do imidacloprid e lambdacyhalothrin no controle do psilídeo (Triozoida sp.), em goiabeira. Revista Brasileira de Fruticultura, Cruz das Almas, v.21, n.3, p.385-387, 1999.

HASSAN, S.A.; BIGLER, F.; BOGENSCHUTZ, H.; BOLLER, E.; BRUN, J.; CALIS, J.N.M.; COREMANS-PELSENEER, J.; DUSO, C.; GROVE, A.; HEIMBACH, U.; HELYER, N.; HOKKANEN, H.; LEWIS, G.B.; MANSOUR, F.; MORETH, L.; POLGAR, L.; SAMSOE-PETERSEN, L.; SAUPHANOR, B.; STAUBLI,A.; STERK, G.; VAINIO, A.; VEIRE, M. van de; VIGGIANI, G.; VOGT, H. Results of the six pesticide testing programme of the IOBC/WPRS-Working group pesticides and beneficial organisms. Entomophaga, Paris, v.39, n.1, p.107-119, 1994.

KIILL, L.H.P.; LIMA, P.C.F. Estimativa de área mínima de amostragem para levantamento de plantas invasoras em frutíferas irrigadas. Científica, Jabotical, v.29, n.1/2, p. 95-102, 2001:

LEMOS, R.N.S.; ARAÚJO, J.R.G.; SILVA, E.A. da; SALLES, J.R. de J. Ocorrência e danos causados por Triozoida sp. (Hemiptera: Psyllidae) em goiabeiras no município de Itapecuru-Mirim-MA. Pesquisa em Foco, São Luiz, v.8, n.11, p.165-168, 2000.

MARICONE, F.A..M.; SOUBIHE SOBRINHO, J. Contribuição para o conhecimento de alguns insetos que depredam a goiabeira (Psidium guajava L.). Piracicaba: ESALQ, Instituto de Genética, 1961, 57p.

MENEZES JÚNIOR, A. de O.; PASINI, A. Parasitóides (Hymenoptera: Chalcidoidea) associados a Triozoida limbata (Enderlein) (Hemiptera: Psyllidae) sobre goiabeira, Psidium guajava L. (Myrtaceae), na região norte do Paraná. In: SIMPÓSIO DE CONTROLE BIOLÓGICO, 7., 2001, Poços de Caldas. Resumos... Poços de Caldas: Universidade Federal de Lavras/Embrapa Centro Nacional de Pesquisa de Milho e Sorgo, 2001. p. 344.

NAKANO, O.; SILVEIRANETO S. Contribuição ao estudo de Triozoida sp. Crawf., praga da goiabeira. Ciência e Cultura, São Paulo, v.20, n.2, p.263-264, 1968.

SOUZA, S.S.P. de; SILVA-FILHO, R.; BARBOSA, R.C.; SILVA, A.L. da; AZEVEDO, O.R.F.; MONTEIRO JUNIOR, A.F. The occurence and biological aspects of the jumping plant lice (Homoptera: Psylidae) on guava plants (Psidium guayava) and 'sombreiro' trees (Victoria fairchildiana) in the State of Rio de Janeiro, Brazil. In: INTERNATIONAL CONGRESS OF ENTOMOLOGY, 21., 2000, Foz do Iguassu. Abstracts... Foz do Iguassu: Embrapa Soja, 2000. v.1, p.287. 